\title{
Effect of Early Enteral Nutrition Support Combined with Chemotherapy on Related Complications and Immune Function of Patients after Radical Gastrectomy
}

\author{
Jing Wang, ${ }^{1}$ Lei Wang, ${ }^{2}$ Min Zhao, ${ }^{3}$ Xiaoxia Zuo, ${ }^{1}$ Wenhua Zhu, ${ }^{4}$ Keying Cui, ${ }^{5}$ Xu Yan, ${ }^{1}$ \\ and Xiaofei Liu $\mathbb{D D}^{6}$ \\ ${ }^{1}$ Department of Clinical Nutrition, The 8th Medical Center of Chinese PLA General Hospital, Beijing 100091, China \\ ${ }^{2}$ Department of Clinical Nutrition, The First Affiliated Hospital, Zhejiang University School of Medicine, Hangzhou 310003, \\ Zhejiang Province, China \\ ${ }^{3}$ Department of Clinical Nutrition, The 6th Medical Center of Chinese PLA General Hospital, Beijing 100048, China \\ ${ }^{4}$ Department of Oncology, The 8th Medical Center of Chinese PLA General Hospital, Beijing 100091, China \\ ${ }^{5}$ Department of General Surgery, The 8th Medical Center of Chinese PLA General Hospital, Beijing 100091, China \\ ${ }^{6}$ Department of Nuclear Medicine, The 8th Medical Center of Chinese PLA General Hospital, Beijing 100091, China
}

Correspondence should be addressed to Xiaofei Liu; liuxiaofei@309yy.org.cn

Received 21 December 2021; Revised 4 January 2022; Accepted 10 January 2022; Published 27 January 2022

Academic Editor: Bhagyaveni M.A

Copyright (c) 2022 Jing Wang et al. This is an open access article distributed under the Creative Commons Attribution License, which permits unrestricted use, distribution, and reproduction in any medium, provided the original work is properly cited.

Objective. The purpose was to analyze the effect of early enteral nutrition (EEN) support combined with chemotherapy on related complications and immune function in patients after radical gastrectomy. Methods. 80 patients with gastric cancer treated in our hospital from March 2019 to March 2020 were selected as the research objects and divided into the experimental group and control group according to the random number table, with 40 cases in each group. The control group received chemotherapy only after surgery, while the experimental group received EEN on this basis. The total protein (TP), transferrin (TF), albumin (ALB), immune cells, and other indexes were measured in the two groups before and after treatment to analyze the effect of different treatment methods on the complications and immune function of patients after radical gastrectomy. Results. There were no significant differences in gender ratio, average age, average BMI, pathological types, disease staging, and residence between the two groups $(P>0.05)$. The exhaust recovery time, total gastric tube drainage, fluid intake time, and hospitalization time in the experimental group were significantly lower than those in the control group $(P<0.05)$. There were no significant differences in the TP, TF, and ALB levels between the two groups before treatment $(P>0.05)$, and the TP, TF, and ALB levels in the experimental group were significantly higher than those in the control group after treatment $(P<0.05)$. The $\mathrm{CD} 4^{+} / \mathrm{CD}^{+}, \mathrm{CD}^{+}$, and $\mathrm{CD} 4^{+}$ levels in the experimental group after treatment were significantly higher than those in the control group $(P<0.001)$. After treatment, the growth hormone levels in both groups significantly increased $(P<0.001)$, and the growth hormone level in the experimental group was significantly higher than that in the control group $(P<0.001)$. There was no significant difference in the KPS scores between the two groups before treatment $(P>0.05)$, and the KPS score in the experimental group was significantly higher than that in the control group after treatment $(P<0.001)$. The incidence of postoperative complications in the experimental group was significantly lower than that in the control group $(P<0.05)$. Conclusion. EEN combined with chemotherapy is a reliable method to improve the immune function of patients after radical gastrectomy for gastric cancer, which plays an important role in improving the physical state of patients and reducing the incidence of complications. Therefore, its further research will help to establish a better treatment plan for such patients. 


\section{Introduction}

Gastric cancer is a common digestive tract malignant tumor disease with a high incidence worldwide [1]. Radical gastrectomy is currently the preferred treatment method for gastric cancer. However, different damage to the gastrointestinal and immune functions of patients will occur under the influence of surgery and tumor consumption, resulting in malnutrition. In addition, gastric cancer patients often need adjuvant chemotherapy after surgery, but the adverse reactions caused by chemotherapy will affect the treatment compliance of patients to a certain extent, increasing the treatment pain and affecting the gastrointestinal function [2-4]. Impaired gastrointestinal absorption function can affect the intake of nutrients, reduce immunity, increase the risk of postoperative infection, and affect prognosis. Therefore, how to improve the gastrointestinal and immune functions of patients after surgery and timely correct malnutrition has become an urgent problem for doctors. EEN has gained extensive concern because studies have confirmed that EEN can effectively reduce the nutritional intake of patients after surgery. However, there is no unified understanding of the effect of EEN on the related complications and immune function of patients after radical gastrectomy [5]. Based on this, this study aims to further explore the effect of EEN support combined with chemotherapy on related complications and immune function of patients after radical gastrectomy and provide a basis for clinical applications, summarized as follows.

\section{Materials and Methods}

2.1. General Information. 80 patients with gastric cancer treated in our hospital from March 2019 to March 2020 were selected as the research objects and divided into the experimental group and control group according to the random number table, with 40 cases in each group. The study was conducted in accordance with the Declaration of Helsinki (as revised in 2013) [6].

2.2. Inclusion Criteria. The inclusion criteria were as follows: (1) the patients were diagnosed with gastric cancer after imaging examinations such as gastroscopy and CT and confirmed by histopathological examination, with the clinical symptoms such as fatigue, anorexia, upper abdominal pain, hematemesis, and melena; (2) the patients received radical gastrectomy (distal gastrectomy, total gastrectomy, and proximal gastrectomy) and had normal intestinal function before surgery; and (3) the patients did not take immunosuppressants recently.

2.3. Exclusion Criteria. The exclusion criteria were as follows: (1) the patients were complicated with immunodeficiency, liver and kidney dysfunction, and endocrine diseases before surgery; (2) the patients received parenteral or enteral nutrition treatment, accompanied by severe gastrointestinal dysfunction; (3) the patients had tumor distant metastasis; and (4) the patients were allergic to nutrient solution.

2.4. Methods. Patients in the control group received chemotherapy after surgery, including tetrahydrofolic acid, oxaliplatin, and fluorouracil. $400 \mathrm{mg} / \mathrm{m}^{2}$ of tetrahydrofolate acid (SFDA approval no. H15021455; manufacturer: Chifeng Mengxin Pharmaceutical Co., Ltd.; specification: $15 \mathrm{mg} / \mathrm{s}$ ) was intravenously injected to the patients on the 1st day. Oxaliplatin (SFDA approval no. H20143023; manufacturer: Hainan Jinrui Pharmaceutical Co., Ltd.; specification: $50 \mathrm{mg}$ ) was intravenously injected on the 1st day, with $85 \mathrm{mg} / \mathrm{m}^{2}$ dripping for more than 2 hours. Fluorouracil injection (manufacturer: Shanghai Xudong Haipu Pharmaceutical Co., Ltd.; NMPA approval no. $\mathrm{H} 31020593$; specification: $10 \mathrm{ml}: 0.25 \mathrm{~g}$ ) was injected on the $2 \mathrm{nd}$ day, with a single dose of $10-20 \mathrm{mg} / \mathrm{kg}$ each day according to the patients' body weight and 2 weeks as a cycle.

The experimental group received EEN support based on the treatment of the control group. The nasointestinal tube was placed at $30-40 \mathrm{~cm}$ below the jejunal anastomosis, and $0.9 \%$ and $500 \mathrm{ml} \mathrm{NaCl}$ solution was infused in 12-24 hours after surgery. Enteral nutritional emulsion (TP) was infused to the patients at 24 hours after surgery, with the calorie as $30 \mathrm{~mL}(30 \mathrm{kcaD} / \mathrm{kg} \cdot \mathrm{d})$ and the nutritional components including saturated fatty acid (1.6\%), fat (3.4\%), protein (3.8\%), unsaturated fatty acid (1.3\%), carbohydrate $(13.8 \%)$, medium-chain triglyceride $(1.2 \%)$, and sugar $(0.5 \%)$. The emulsion was continuously infused for 7 days, following the principle of "first slow and then fast, and first dilute and then thick." The dosage and infusion rate were gradually adjusted according to the patients' tolerance, from half amount to full amount. After oral diet, patients could gradually reduce the intake of enteral nutrition and properly supplement electrolytes, vitamins, and trace elements. The temperature of nutrient solution was controlled at about $39^{\circ} \mathrm{C}$ to avoid cold stimulation which caused intestinal spasm, leading to abdominal pain and diarrhea $[7,8]$.

2.5. Observation Indexes. The exhaust recovery time, total gastric tube drainage, fluid intake time, and hospitalization time were recorded and compared between the two groups.

$5 \mathrm{ml}$ of fasting venous blood was collected from both groups before and after treatment, and the upper serum was taken after centrifugation. An automatic biochemical analyzer (manufacturer: Beijing Perlong Technology Co., Ltd.; model: 600A/B) was used to detect the nutritive indexes (TP, $\mathrm{TF}$, and ALB) in both groups before and after treatment.

A flow cytometer (manufacturer: Shanghai Huanxi Medical Devices Co., Ltd.; model: XTG-1600E) was used to detect the $\mathrm{CD}^{+}, \mathrm{CD}^{+}$, and $\mathrm{CD}^{+} / \mathrm{CD}^{+}$levels.

Radioimmunoassay was used to detect the growth hormone level in both groups after treatment. The kits were purchased from Shanghai Hengyuan Biotechnology Co., Ltd.

Karnofsky score (KPS) [9] was used to evaluate the physical state in both groups before and after treatment. The total score of the scale was 100 points, and the score $\geq 80$ indicated that the patients could completely take care of themselves, 50-79 indicated that the patients could partially take care of themselves, and $\leq 49$ indicated that the patients could not take care of themselves.

The incidence of clinical complications during treatment was recorded and compared between the two groups, including diarrhea, abdominal pain, and phlebitis. 
2.6. Statistical Methods. All the experimental data were statistically analyzed and processed by SPSS 21.0 software, and GraphPad Prism 7 (GraphPad Software, San Diego, USA) was used to draw pictures of the data. The count data were tested by $X^{2}$, expressed by $n(\%)$, and the measurement data were measured by $t$-test, expressed by $(-x \pm s)$. The difference was statistically significant when $P<0.05$.

\section{Results}

3.1. Comparison of Baseline Data between the Two Groups. There were no significant differences in gender ratio, average age, average BMI, pathological types, disease staging, and residence between the two groups $(P>0.05)$, indicating comparability, as shown in Table 1.

3.2. Comparison of Postoperative Recovery between the Two Groups. The exhaust recovery time, total gastric tube drainage, fluid intake time, and hospitalization time in the experimental group were significantly lower than those in the control group $(P<0.05)$, as shown in Table 2 .

3.3. Comparison of Nutritive Indexes before and after Treatment between the Two Groups. There were no significant differences in the TP, TF, and ALB levels between the two groups before treatment $(P>0.05)$, and the TP, TF, and ALB levels in the experimental group were significantly higher than those in the control group after treatment $(P<0.05)$, as shown in Table 3.

3.4. Comparison of Immune Indexes after Treatment between the Two Groups. The $\mathrm{CD} 4^{+} / \mathrm{CD}^{+}, \mathrm{CD}^{+}$, and $\mathrm{CD} 4^{+}$levels in the experimental group after treatment were significantly higher than those in the control group $(P<0.05)$, as shown in Table 4.

3.5. Comparison of Growth Hormone Levels before and after Treatment between the Two Groups. After treatment, the growth hormone levels in both groups significantly increased $(P<0.05)$, and the growth hormone level in the experimental group was significantly higher than that in the control group $(P<0.05)$, as shown in Figure 1 .

3.6. Comparison of KPS Scores before and after Treatment between the Two Groups. There was no significant difference in the KPS scores between the two groups before treatment $(P>0.05)$, and the KPS score in the experimental group was significantly higher than that in the control group after treatment $(P<0.05)$, as shown in Figure 2.

3.7. Comparison of the Incidence of Complications between the Two Groups. The incidence of postoperative complications in the experimental group was significantly lower than that in the control group $(P<0.05)$, as shown in Table 5 .

\section{Discussion}

Gastric cancer can occur in any part of the stomach. The pathogenesis is that the mutation of a single gene results in the exponential growth of gastric cancer cells and decreased body's immune function or immune escape effect of gastric cancer cells so that the body's immune system cannot kill gastric cancer cells. At this time, cancer cells will break through the basal layer, and distant metastasis occurs through the lymphatic and blood systems, thereby affecting the normal diet and digestion and hindering the absorption of nutrients. Radical gastrectomy will cause stress reactions in patients, which accelerate the catabolism and aggravate the degree of malnutrition, leading to decreased immune function, postoperative complications, and poor prognosis [10-12]. Chemotherapy is a common auxiliary method for patients with gastric cancer after surgery, which can inhibit the subclinical disease in patients to a certain extent, reduce the activity of tumor cells, minimize the chance of tumor cell proliferation after surgery, and reduce the possibility of recurrence. In addition, chemotherapy can also reduce the adhesion of tumor tissue, decrease the clinical tumor stages, and improve the success rate of surgical resection [13-15]. However, chemotherapy will have severe strong gastrointestinal reactions, myelosuppression, alopecia, and other complications, increasing the pain of treatment and seriously affecting the life quality [16]. In addition, investigations have found that most patients with gastric cancer have varying degrees of malnutrition and immunosenescence symptoms before admission due to vomiting, tumor consumption, and other factors, which will further deteriorate after a series of clinical treatments such as surgery and chemotherapy. Therefore, how to implement nutritional support and treatment for patients after radical surgery is crucial to their rehabilitation, the improvement of immune function, and the reduction of related complications [17-19].

EEN can not only provide nutrition for patients but also reduce oxidative stress, protect the gastrointestinal structure and function, and reduce inflammatory response, so as to maintain the functions of organs, tissues, and cells, improve nutrition absorption rate, enhance immune function, and promote rehabilitation, which has been confirmed in diseases such as ischemic stroke, acute pancreatitis, and esophagus cancer $[20,21]$. In this study, the patients in the experimental group received nutritional support with a nasointestinal tube which is simple and safe, with little impact on the patient comfort. In addition, the anastomotic stoma was avoided during feeding to avoid the food stimulation to the stoma and improve the nutritional absorption. After treatment, the exhaust recovery time, total gastric tube drainage, fluid intake time, and hospitalization time in the experimental group were significantly lower than those in the control group $(P<0.001)$, indicating that EEN combined with chemotherapy can significantly shorten the recovery time of patients after surgery, which is more effective than chemotherapy alone. The immune function is mainly exerted through cellular immunity, in which $\mathrm{T}$ lymphocytes are the main effector cells and can directly reflect the body 
TABLE 1: Comparison of baseline data between the two groups.

\begin{tabular}{|c|c|c|c|c|}
\hline Items & Experimental group $(n=40)$ & Control group $(n=40)$ & $\chi^{2} / t$ & $P$ \\
\hline Gender & & & 0.205 & 0.651 \\
\hline Male & $22(59.46 \%)$ & $24(64.86 \%)$ & & \\
\hline Female & $18(48.65 \%)$ & $16(43.24 \%)$ & & \\
\hline Average age (years) & $43.71 \pm 4.32$ & $43.59 \pm 4.36$ & 0.124 & 0.902 \\
\hline Average BMI $\left(\mathrm{kg} / \mathrm{m}^{2}\right)$ & $21.65 \pm 1.26$ & $21.57 \pm 1.28$ & 0.282 & 0.779 \\
\hline \multicolumn{5}{|l|}{ Pathological types } \\
\hline Mucinous carcinoma & $4(10.00 \%)$ & $6(15.00 \%)$ & 0.457 & 0.499 \\
\hline Medium differentiated adenocarcinoma & $13(32.50 \%)$ & $12(30.00 \%)$ & 0.058 & 0.809 \\
\hline Poorly differentiated adenocarcinoma & $6(15.00 \%)$ & $8(20.00 \%)$ & 0.346 & 0.556 \\
\hline Highly differentiated adenocarcinoma & $17(42.50 \%)$ & $14(35.00 \%)$ & 0.474 & 0.491 \\
\hline \multicolumn{5}{|l|}{ Disease staging } \\
\hline I & $11(27.50 \%)$ & $13(32.50 \%)$ & 0.238 & 0.626 \\
\hline II & $17(42.50 \%)$ & $14(35.00 \%)$ & 0.474 & 0.491 \\
\hline III & $12(30.00 \%)$ & $13(32.50 \%)$ & 0.058 & 0.809 \\
\hline Residence & & & 0.802 & 0.370 \\
\hline Urban area & $19(47.50 \%)$ & $23(57.50 \%)$ & & \\
\hline Rural area & $21(52.50 \%)$ & $17(42.50 \%)$ & & \\
\hline
\end{tabular}

TABLE 2: Comparison of postoperative recovery between the two groups ( $x \pm s)$.

\begin{tabular}{lccccc}
\hline Group & $n$ & Exhaust recovery time $(\mathrm{h})$ & Total gastric tube drainage $(\mathrm{mL})$ & Fluid intake time $(\mathrm{d})$ & Hospitalization time $(\mathrm{d})$ \\
\hline Experimental group & 40 & $81.94 \pm 13.27$ & $263.81 \pm 38.92$ & $5.42 \pm 1.06$ & $12.52 \pm 4.82$ \\
Control group & 40 & $91.72 \pm 12.65$ & $374.81 \pm 36.57$ & $7.32 \pm 1.21$ & 7.24 \\
$t$ & & 3.374 & 13.145 & $<.470$ & 4.34 \\
$P$ & $<0.05$ & $<0.001$ & $<0.001$ & $<0.001$ \\
\hline
\end{tabular}

TABLe 3: Comparison of nutritive indexes before and after treatment between the two groups ( $x \pm s$ ).

\begin{tabular}{|c|c|c|c|c|c|c|c|}
\hline \multirow{2}{*}{ Group } & \multirow{2}{*}{$n$} & \multicolumn{2}{|c|}{$\mathrm{TP}\left(\mathrm{g} \cdot \mathrm{L}^{-1}\right)$} & \multicolumn{2}{|c|}{$\mathrm{TF}\left(\mathrm{mg} \cdot \mathrm{L}^{-1}\right)$} & \multicolumn{2}{|c|}{$\operatorname{ALB}(g / L)$} \\
\hline & & Before treatment & After treatment & Before treatment & After treatment & Before treatment & After treatment \\
\hline Experimental group & 40 & $58.21 \pm 6.25$ & $66.87 \pm 5.82$ & $127.69 \pm 8.48$ & $153.27 \pm 6.79$ & $26.51 \pm 2.31$ & $40.26 \pm 3.18$ \\
\hline Control group & 40 & $58.27 \pm 6.34$ & $63.18 \pm 5.42$ & $127.73 \pm 8.53$ & $146.72 \pm 6.54$ & $26.47 \pm 2.36$ & $34.52 \pm 3.14$ \\
\hline$t$ & & 0.043 & 2.934 & 0.021 & 4.394 & 0.077 & 8.123 \\
\hline$P$ & & 0.966 & $<0.05$ & 0.983 & $<0.001$ & 0.939 & $<0.001$ \\
\hline
\end{tabular}

TABLE 4: Comparison of immune indexes after treatment between the two groups $\left({ }^{-} x \pm s\right)$.

\begin{tabular}{lcccc}
\hline Group & $n$ & $\mathrm{CD}^{+} / \mathrm{CD}^{+}$ & $\mathrm{CD}^{+}(\%)$ & $\mathrm{CD}^{+}(\%)$ \\
\hline Experimental group & 40 & $1.65 \pm 0.42$ & $64.32 \pm 4.29$ & $43.18 \pm 3.19$ \\
Control group & 40 & $1.32 \pm 0.34$ & $58.63 \pm 4.38$ & $38.71 \pm 3.85$ \\
$t$ & & 3.862 & 5.870 & 5.654 \\
$P$ & & $<0.001$ & $<0.001$ & $<0.001$ \\
\hline
\end{tabular}

immune condition in the perioperative period $[22,23]$. EEN contributes to the recovery of intestinal function and maintains the integrity of the intestinal mucosal structure and function. This study showed that the $\mathrm{CD} 4^{+} / \mathrm{CD}^{+}$, $\mathrm{CD}^{+}$, and $\mathrm{CD}^{+}$levels in the experimental group after treatment were significantly higher than those in the control group $(P<0.001)$. Tai et al. [24] pointed out that, after the patients with stage II gastric cancer received EEN based on the radical gastrectomy, the $\mathrm{CD}^{+}$and $\mathrm{CD} 4^{+}$levels of patients were $62.47 \pm 3.64 \%$ and $42.18 \pm 3.81 \%$, which were significantly higher than $58.62 \pm 3.49 \%$ and $36.34 \pm 3.24 \%$ before treatment, suggesting that EEN can significantly improve the immune function of patients with gastric cancer after radical gastrectomy. In addition, the study also found that the incidence of postoperative complications in the experimental group was significantly lower than that in the control group $(P<0.05)$. It was speculated that EEN preparations were rich in nutrients such as fat, amino acids, and vitamins, which could enhance the blood flow in the gastrointestinal tract of patients, promote digestive movement and the secretion of related hormones, and provide the body with the required energy supply, thus improving the patients' own immunity and greatly reducing the risk of postoperative complications. Since this study is a singlecenter and small-sample test, it is not sufficient to assess the differences in postoperative immune function and complications. In order to reduce bias, the selected patients were screened, and those with endocrine diseases and distant 


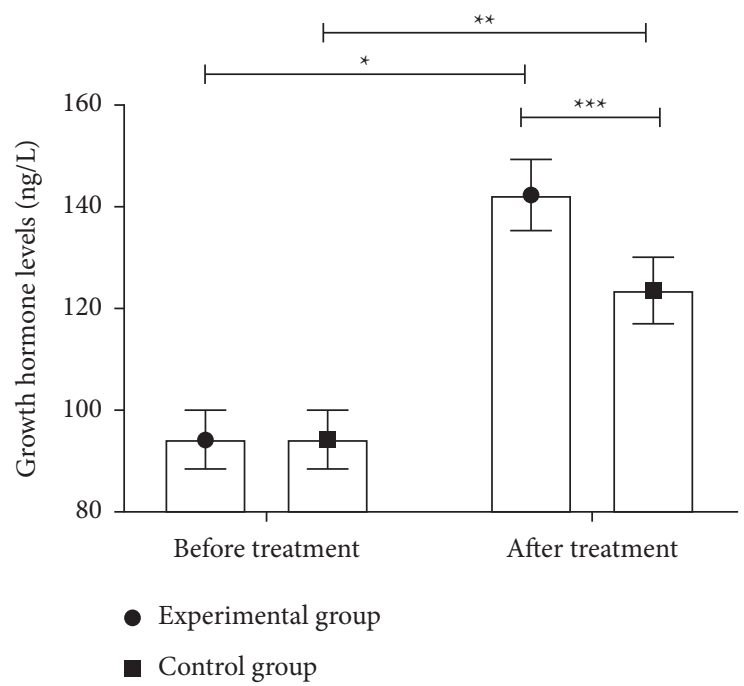

FIGURE 1: Comparison of growth hormone levels before and after treatment between the two groups ( $-x \pm s)$. Note: the abscissa represents before treatment and after treatment, and the ordinate represents the growth hormone level (ng/L). The growth hormone levels in the experimental group before and after treatment were $94.25 \pm 5.84 \mathrm{ng} / \mathrm{L}$ and $142.37 \pm 6.94 \mathrm{ng} / \mathrm{L}$, respectively. The growth hormone levels in the control group before and after treatment were $94.46 \pm 5.69 \mathrm{ng} / \mathrm{L}$ and $123.54 \pm 6.53 \mathrm{ng} / \mathrm{L}$, respectively. ${ }^{*}$ A significant difference in the growth hormone levels of the experimental group before and after treatment $(t=33.553, P<0.001)$. ${ }^{* *} \mathrm{~A}$ significant difference in the growth hormone levels of the control group before and after treatment $(t=21.235, P<0.001) .{ }^{* * *} \mathrm{~A}$ significant difference in the growth hormone levels between the two groups after treatment $(t=12.498, P<0.001)$.

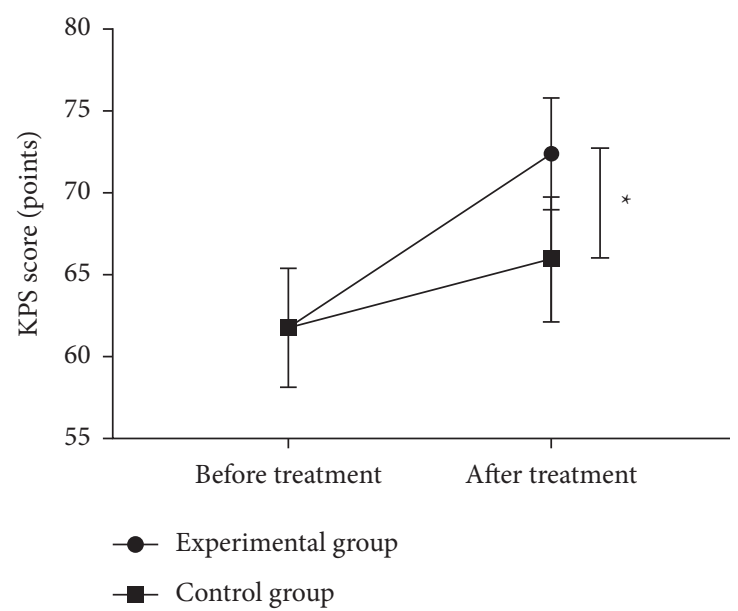

FIGURE 2: Comparison of KPS scores before and after treatment between the two groups $(-x \pm s)$. Note: the abscissa represents before treatment and after treatment, and the ordinate represents the KPS score (points). The KPS scores in the experimental group before and after treatment were $61.83 \pm 3.54$ and $72.42 \pm 3.44$, respectively. The KPS scores in the control group before and after treatment were $61.79 \pm 3.63$ and $65.96 \pm 3.81$, respectively. ${ }^{*}$ A significant difference in the KPS scores between the two groups after treatment $(t=7.959, P<0.001)$.

TABLE 5: Comparison of the incidence of complications between the two groups $(n(\%))$.

\begin{tabular}{|c|c|c|c|c|c|}
\hline Group & $n$ & Diarrhea & Abdominal pain & Phlebitis & Total incidence \\
\hline Experimental group & 40 & $2(5.00 \%)$ & $1(2.50 \%)$ & $1(2.50 \%)$ & $10.00 \%(4 / 40)$ \\
\hline Control group & 40 & $4(10.00 \%)$ & $4(10.00 \%)$ & $3(7.50 \%)$ & $27.50 \%(11 / 40)$ \\
\hline$X^{2}$ & & & & & 4.021 \\
\hline$P$ & & & & & $<0.05$ \\
\hline
\end{tabular}

metastasis of tumors were excluded. Therefore, this experiment cannot comprehensively describe the clinical symptoms of patients, but indicated the direction for the subsequent research studies.
In conclusion, EEN combined with chemotherapy is a reliable method to improve the nutritional status and immune function of patients with gastric cancer after radical gastrectomy. This treatment method greatly shortens the 
postoperative recovery time of patients and reduces the incidence of postoperative complications, which has a high clinical application value.

\section{Data Availability}

The data used to support the findings of this study are available from the corresponding author upon reasonable request.

\section{Conflicts of Interest}

The authors declare no conflicts of interest.

\section{Authors' Contributions}

Jing Wang and Lei Wang contributed equally to this work.

\section{References}

[1] K. C. Greathouse, K. T. Sakellaris, D. Tumin et al., "Impact of early initiation of enteral nutrition on survival during pediatric extracorporeal membrane oxygenation," Journal of Parenteral and Enteral Nutrition, vol. 42, no. 1, pp. 205-211, 2018.

[2] H. Beverly, R. Walker, U. Andrew et al., "Standards for nutrition support: adult hospitalized patients," Nutrition In Clinical Practice: Official Publication of the American Society for Parenteral and Enteral Nutrition, vol. 33, no. 6, pp. 906920, 2018.

[3] A. Wong, M. D. Banks, and J. D Bauer, "A survey of home enteral nutrition practices and reimbursement in the asia pacific region," Nutrients, vol. 10, no. 2, 2018.

[4] Standards for Nutrition Support, "Adult hospitalized patients," Nutrition in Clinical Practice, vol. 33, no. 6, pp. 906-920, 2018.

[5] B. Parent, M. Seaton, and G. E. Grant, "Biochemical markers of nutrition support in critically ill trauma victims," JPEN Journal of Parenteral and Enteral Nutrition, vol. 42, no. 2, pp. 335-342, 2018.

[6] World Medical Association, "World medical association declaration of Helsinki: ethical principles for medical research involving human subjects," JAMA, vol. 310, no. 20, pp. 2191-2194, 2013.

[7] Q. S. Yu, Z. Zheng, H. Peng, Y Shen, J. D Liu, and F. H Zhou, "Effect of qihuang decoction combined with enteral nutrition on postoperative gastric cancer of nutrition and immune function," Evidence-based Complementary and Alternative Medicine :eCAM, vol. 2020, Article ID 1795107, 10 pages, 2020.

[8] Y. Ye, Y. Xu, Q. Fu et al., "Enteral nutrition support does not improve PNI in radiotherapy patients with locally advanced esophageal cancer," Nutrition and Cancer, vol. 71, no. 2, pp. 223-229, 2019.

[9] N. Amy, "Help for parents providing home enteral nutrition," Journal of health visiting, vol. 7, no. 5, pp. 210-211, 2019.

[10] K. Allen and L. Hoffman, "Enteral nutrition in the mechanically ventilated patient.nutrition in clinical practice," Nutrition in Clinical Practice, vol. 34, no. 4, pp. 540-557, 2019.

[11] S.-A. Lui, W. B. Tan, B. Choo Tai et al., "Predictors of survival outcome following radical gastrectomy for gastric cancer," ANZ Journal of Surgery, vol. 89, no. 1-2, pp. 84-89, 2019.
[12] B. Sato, M. Kanda, C. Tanaka et al., "Significance of preoperative systemic inflammation score in short-term and longterm outcomes of patients with pathological T2-4 gastric cancer after radical gastrectomy," World Journal of Surgery: Official Journal of the Societe Internationale de Chirurgie, Collegium Internationale Chirurgiae Digestivae, and of the International Association of Endocrine Surgeons, vol. 42, no. 10 , pp. 3277-3285, 2018.

[13] X. Xia, Z. Zhang, J. Xu, G. Zhao, and F. Yu, "Comparison of postoperative lymphocytes and interleukins between laparoscopy-assisted and open radical gastrectomy for early gastric cancer," Journal of International Medical Research, vol. 47, no. 1, pp. 303-310, 2019.

[14] C.-L. Zhuang, X. Shen, Y.-Y. Huang et al., "Myosteatosis predicts prognosis after radical gastrectomy for gastric cancer: a propensity score-matched analysis from a large-scale cohort," Surgery, vol. 166, no. 3, pp. 297-304, 2019.

[15] H. Okabe, K. Obama, S. Tsunoda et al., "Feasibility of robotic radical gastrectomy using a monopolar device for gastric cancer," Surgery Today, vol. 49, no. 10, pp. 820-827, 2019.

[16] I. G. Kwon, T. Son, H.-I. Kim, and W. J. Hyung, "Fluorescent lymphography-guided lymphadenectomy during robotic radical gastrectomy for gastric cancer," JAMA surgery, vol. 154, no. 2, pp. 150-158, 2019.

[17] Q.-Y. Chen, Q. Zhong, Z.-Y. Liu et al., "Does noncompliance in lymph node dissection affect oncological efficacy in gastric cancer patients undergoing radical gastrectomy?" Annals of Surgical Oncology, vol. 26, no. 6, pp. 1759-1771, 2019.

[18] Z.-F. Zheng, J. Lu, J.-W. Xie et al., "Preoperative skeletal muscle index vs the controlling nutritional status score: which is a better objective predictor of long-term survival for gastric cancer patients after radical gastrectomy?" Cancer Medicine, vol. 7, no. 8, pp. 3537-3547, 2018.

[19] X.-L. Meng and T. Li, "Curative effect of digestive tract reconstruction after radical gastrectomy for distal gastric cancer," Journal of Laparoendoscopic and Advanced Surgical Techniques, Part A, vol. 28, no. 11, pp. 1294-1297, 2018.

[20] K. Takahashi, S. Mine, R. Toihata, A. Okamura, Y. Imamura, and M. Watanabe, "Ivor-Lewis esophagectomy for patients with squamous cell carcinoma of the thoracic esophagus with a history of total pharyngolaryngectomy," Esophagus, vol. 16, no. 4, pp. 382-385, 2019.

[21] C. Katada, T. Yokoyama, T. Yano et al., "Association between macrocytosis and metachronous squamous cell carcinoma of the esophagus after endoscopic resection in men with early esophageal squamous cell carcinoma," Esophagus : Official Journal of the Japan Esophageal Society, vol. 17, pp. 149-158, 2020.

[22] M. Kanda, C. Tanaka, D. Kobayashi et al., "Preoperative albumin-bilirubin grade predicts recurrences after radical gastrectomy in patients with pT2-4 gastric cancer," World Journal of Surgery, vol. 42, no. 3, pp. 773-781, 2018.

[23] X.-Y. Chen, S.-L. Wang, L.-L. Ma et al., "Impact of visceral fat on surgical complications and long-term survival of patients with gastric cancer after radical gastrectomy," European Journal of Clinical Nutrition, vol. 72, no. 3, pp. 436-445, 2018.

[24] B. Tai, Y. Choo, W. Peng, A. Shabbir, J. So, and S. Lui, "Predictors of survival outcome following radical gastrectomy for gastric cancer," ANZ Journal of Surgery, vol. 89, no. 1/2, pp. 84-89, 2019. 